\title{
Caracterización de la retinopatía diabética en un programa de tamización en Medellín, Colombia, en el año 2018
}

\section{Characterization of diabetic retinopathy in a screening program in Medellin, Colombia, in 2018}

\author{
José P. Vivas-Giraldo ${ }^{1,2 *}$ y Juan D. Bravo-Acosta ${ }^{1,3}$ \\ ${ }^{1}$ Servicio de Oftalmología, Universidad de Antioquia, Antioquia; ${ }^{2}$ Clínica Oftalmológica del Café, Manizales, Caldas; ${ }^{3}$ Servicio de Retina y Vítreo, \\ Hospital San Vicente Fundación, Medellín, Antioquia. Colombia
}

\section{Resumen}

Objetivo: El propósito de este estudio es determinar la prevalencia de la retinopatía diabética en pacientes diabéticos de un programa de tamización del Hospital San Vicente Fundación de Medellín (Colombia), describir las características sociodemográficas y el tipo de retinopatía diabética, y evaluar el porcentaje de pacientes con retinopatía que amenaza la visión. Método: Estudio descriptivo, transversal, en el que se evaluó una base de datos de pacientes diabéticos de todas las edades y ambos sexos que acudieron a la campaña «No más ciegos por diabetes» del Hospital San Vicente Fundación de Medellín, realizada en el año 2018. Resultados: Se evaluaron 610 pacientes diabéticos de la campaña de 2018. La mayoría tenían diabetes tipo 2, en total 528 (74,8\%). De los pacientes evaluados, en 115 se encontró retinopatía, para una prevalencia del 18,8\%. El tipo proliferativo se encontró en 17 (14,7\%) pacientes y el tipo no proliferativo fue el más frecuente, con 98 pacientes (85,2\%). El subgrupo de retinopatía que amenaza la visión estaba constituido por 48 (7,8\%) pacientes, de los cuales en $7(14,5 \%)$ no se había hecho el diagnóstico previamente. Conclusión: La prevalencia de la retinopatía diabética en la población estudiada es comparable a la encontrada en algunos de los estudios realizados en otros países de América Latina. Este trabajo aporta datos epidemiológicos locales relevantes sobre esta enfermedad y refuerza la importancia de continuar realizando campañas de tamización para prevenir los casos de ceguera gracias a un diagnóstico y un tratamiento tempranos.

Palabras clave: Diabetes. Retinopatía diabética. Prevalencia. Edema macular. Tamización. Clasificación.

\section{Abstract}

Purpose: To identify the prevalence of diabetic retinopathy in diabetic patients of a screening program of the Hospital San Vicente Fundación in Medellin (Colombia), to describe the sociodemographic characteristics and the type of diabetic retinopathy and to evaluate the percentage of patients with sight-threatening diabetic retinopathy. Method: This is a cross-sectional descriptive study, in which diabetic patients of all ages and both genders were evaluated in the "No more blind from diabetes" campaign of the Hospital San Vicente Fundación in Medellín, conducted in the year 2018. Results: We evaluated 610 diabetic patients from the 2018 campaign. Most of the patients had type 2 diabetes, in total 528 (74.8\%). Of the patients evaluated, retinopathy was found in 115 cases, for a prevalence of $18.8 \%$. Proliferative retinopathy was found in 17 (14.7\%) patients, and non-proliferative retinopathy was the most frequent in 98 patients (85.2\%). The subgroup of sight-threatening

Fecha de recepción: 05-10-2020

Fecha de aceptación: 25-02-2021

DOI: 10.24875/RMO.M21000169
Disponible en internet: 05-05-2021 Rev Mex Oftalmol. 2021;95(3):118-123

www.rmo.com.mx 0187-4519/O 2021 Sociedad Mexicana de Oftalmología. Publicado por Permanyer. Este es un artículo open access bajo la licencia CC BY-NC-ND (http://creativecommons.org/licenses/by-nc-nd/4.0/). 
diabetic retinopathy was made up of 48 (7.8\%) patients, of which the diagnosis had not been made previously in 7 (14.5\%) cases. Conclusions: The prevalence of diabetic retinopathy in the population studied is comparable with some of the studies carried out in some Latin American countries. This work provides relevant local epidemiological data about diabetic retinopathy and reinforces the importance of continuing screening campaigns to prevent cases of blindness with early diagnosis and treatment.

Key words: Diabetes. Diabetic retinopathy. Prevalence. Macular oedema. Screening. Classification.

\section{Introducción}

La diabetes es una enfermedad metabólica crónica compleja asociada a valores elevados de glucosa en sangre que requiere un cuidado médico continuo, con estrategias de reducción del riesgo multifactorial para prevenir las complicaciones macrovasculares y microvasculares a largo plazo. Según la Organización Mundial de la Salud (OMS), su prevalencia es del $8,5 \%$ en las personas mayores de 18 años y anualmente mueren entre 1,6 y 2 millones de personas en el mundo como consecuencia de ella. La prevalencia de la diabetes está aumentando mundialmente y se dice que para el año 2025 este número podría aumentar hasta el doble ${ }^{1}$.

La retinopatía diabética $(\mathrm{RD})$ es una alteración microvascular progresiva que genera isquemia retinal, aumento en la permeabilidad vascular, neovascularización retinal y edema macular ${ }^{2}$. Se ha reportado que uno de cada tres $(34,6 \%)$ pacientes diabéticos tiene algún tipo de $\mathrm{RD}^{3}$. Todos los pacientes diabéticos están en riesgo de $\mathrm{RD}$ y en los países desarrollados esta enfermedad es la principal causa de ceguera en la edad productiva ${ }^{4}$. Así mismo, como va aumentando la prevalencia de la diabetes, se incrementa también la prevalencia de la RD, y la que amenaza la visión también lo hace de forma dramática'.

Es muy importante categorizar, clasificar y estadificar la gravedad de la RD para poder establecer un tratamiento adecuado y ofrecer al paciente un pronóstico preciso según la gravedad de su enfermedad. La clasificación internacional de la RD incluye cinco estadios: 1) sin retinopatía aparente; 2) RD no proliferativa (RDNP) leve; 3) RDNP moderada; 4) RDNP grave; y 5) RD proliferativa (RDP) caracterizada por neovascularización del disco, la retina, el iris o el ángulo camerular, hemorragia vítrea o desprendimiento de retina traccional ${ }^{2}$. El edema macular diabético (EMD) puede estar asociado a cualquier tipo de RD o presentarse de forma aislada².

Existe una subclasificación, denominada RD que amenaza la visión, en la que se incluyen la RDNP grave, la RDP y el EMD 5 . Se ha encontrado que 1 de cada $10(10,2 \%)$ pacientes con RD presenta este tipo que amenaza la visión y que la tendencia va en aumento a medida que aumenta la prevalencia de la diabetes ${ }^{3}$.

Actualmente solo alrededor del $60 \%$ de los pacientes diabéticos tienen tamización anual para $\mathrm{RD}^{1}$, por lo cual las campañas como la que se realiza en el Hospital San Vicente Fundación de la Ciudad de Medellín son de vital importancia para caracterizar a la población local y detectar los casos de RD, sobre todo el grupo de pacientes con RD que amenaza la visión, de manera que se pueda intervenir de forma temprana para reducir los casos de pérdida visual asociados a las complicaciones de esta enfermedad.

El propósito de este estudio fue caracterizar y evaluar la prevalencia de la RD en los pacientes con diagnóstico de diabetes en el Hospital San Vicente Fundación de Medellín en la campaña «No más ciegos por diabetes» de 2018, describir las características sociodemográficas y el tipo de RD en estos pacientes según la clasificación internacional para la gravedad de la RD, y conocer el porcentaje de pacientes en el grupo de RD que amenaza la visión.

\section{Método}

Este es un estudio descriptivo, transversal, en el que se evalúan los datos obtenidos de pacientes diabéticos de todas las edades y ambos sexos que acudieron a la campaña de tamización «No más ciegos por diabetes» realizada en el hospital San Vicente Fundación de la ciudad de Medellín, que se realizó en noviembre de 2018.

En esta campaña, los pacientes diabéticos de varias regiones del departamento de Antioquia acuden espontáneamente una vez al año al hospital y se les toman datos de sus antecedentes y del estado actual de su enfermedad mediante una encuesta, y además se les realiza un examen de fondo de ojo con pupila dilatada, ya sea en lámpara de hendidura con un lente de 90 dioptrías o con oftalmoscopio indirecto por un oftalmólogo designado que registra los hallazgos, clasificando los pacientes como positivos o negativos para RD, y en caso de presentarla se clasifica según la clasificación internacional de la RD y el EMD para definir la 
conducta sobre tratamiento inmediato, diferido 0 simplemente control según el riesgo.

Se excluyeron los pacientes que no tenían la información completa en la base de datos. No se calculó tamaño de muestra ya que esta es por conveniencia e incluye el total de pacientes que cumplen los criterios de elegibilidad.

Se consideraron las siguientes variables cualitativas: sexo, barrio de procedencia, asistencia nueva o repetida a la campaña, conocimiento acerca de la RD, tipo de diabetes, tabaquismo activo, antecedente de hipertensión arterial (HTA), antecedente de dislipidemia, conocimiento sobre la hemoglobina glucosilada (HbA1c), tiempo de la última evaluación por oftalmología, complicaciones asociadas a la diabetes, presencia de RD, tipo de RD, presencia de EMD, diagnósticos asociados y necesidad de tratamiento urgente en la siguiente semana según el tiempo de respuesta de su aseguradora, con apoyo de la trabajadora social. Las variables cuantitativas evaluadas fueron la edad, el tiempo de evolución de la diabetes en años y el último valor de la HbA1c.

El presente estudio es catalogado como investigación sin riesgo según la resolución 8430 de 1993 del Ministerio de Salud de Colombia. Previa socialización del estudio se solicitó su aprobación por parte del comité de ética a la dirección de investigaciones del Hospital San Vicente Fundación. Por tratarse de un estudio transversal de fuentes secundarias no es necesario aplicar consentimiento informado para el uso de la información recolectada durante las jornadas anuales de la campaña "No más ciegos por diabetes». Se guarda la confidencialidad de la información y de la identificación de los pacientes. La información se usará solo con fines de investigación y teniendo respeto por su integridad.

La información se exportó, almacenó y procesó en el programa estadístico SPSS v21®. Las tablas, los gráficos y la presentación del texto se realizaron mediante el procesador de texto de Microsoft Office Word 365.

Las variables cuantitativas se expresan con su mediana y rango intercuartílico $(\mathrm{RIQ})$ debido a su distribución no normal, y las variables cualitativas se muestran en número absoluto y porcentaje.

\section{Resultados}

Se evaluaron 610 registros de una base de datos de pacientes diabéticos de la campaña de 2018. Sus características demográficas se detallan en la tabla 1.
Tabla 1. Características sociodemográficas de la población de estudio

\begin{tabular}{|c|c|c|}
\hline Variable & $n=610$ & Porcentaje \\
\hline Edad en años, media (RIQ) & \multicolumn{2}{|c|}{$60,5(54-69)$} \\
\hline Sexo masculino & 220 & 36 \\
\hline Asistencia por primera vez & 276 & 45 \\
\hline $\begin{array}{l}\text { Conocimiento de la retinopatía } \\
\text { diabética }\end{array}$ & 202 & 33 \\
\hline $\begin{array}{l}\text { Tiempo de diabetes en años, } \\
\text { media (RIQ) }\end{array}$ & \multicolumn{2}{|c|}{$12,5(5-18)$} \\
\hline Pacientes con diabetes tipo 2 & 528 & 74,8 \\
\hline Tabaquismo activo & 44 & 6,2 \\
\hline Hipertensión arterial & 381 & 62,4 \\
\hline Dislipidemia & 380 & 62,2 \\
\hline Conocimiento de $\mathrm{HbA} 1 \mathrm{c}$ & 340 & 56 \\
\hline Valores de Hba1c, media (RI0) & \multicolumn{2}{|c|}{$7,7(6,6-8,4)$} \\
\hline $\begin{array}{l}\text { Complicaciones asociadas a la } \\
\text { diabetes }\end{array}$ & 139 & 22,7 \\
\hline Daño renal & 46 & 7,5 \\
\hline \multicolumn{3}{|l|}{ Tiempo de última evaluación } \\
\hline Menos de 1 año & 312 & 52 \\
\hline Más de 1 año o nunca & 298 & 48 \\
\hline Retinopatía diabética & 115 & 18 \\
\hline Proliferativa & 17 & 14,7 \\
\hline No proliferativa & 98 & 85,2 \\
\hline Leve & 57 & 58,1 \\
\hline Moderada & 33 & 33,6 \\
\hline Grave & 8 & 8,1 \\
\hline Edema macular & 32 & 5,2 \\
\hline Otros diagnósticos & 141 & 23,1 \\
\hline Catarata & 96 & 15,7 \\
\hline Glaucoma & 17 & 2,7 \\
\hline $\begin{array}{l}\text { Degeneración macular asociada } \\
\text { a la edad }\end{array}$ & 19 & 3,1 \\
\hline Otros diagnósticos & 9 & 1,4 \\
\hline Requiere tratamiento urgente & 17 & 2,7 \\
\hline
\end{tabular}

Se encontró un predominio del sexo femenino, con 390 pacientes $(63,9 \%$ ), y la mediana de edad de todos los pacientes fue de 60,5 años (RIQ: 54-69). La 
Tabla 2. Características de la población de pacientes con retinopatía diabética

\begin{tabular}{|l|c|c|c|}
\hline & RDP $n=17(14,7 \%)$ & RDNP $n=98(85,2 \%)$ & $p^{*}$ \\
\hline Tiempo de evolución en años, mediana (RIO) & $22,1(12-29,5)$ & $15,5(8-20,2)$ & $0,027^{\dagger}$ \\
\hline Valor de HbA1c, mediana (RIO) & $8,1(6,8-8,9)$ & $7,6(6,9-8,9)$ & $0,857^{\dagger}$ \\
\hline Daño renal, $\mathrm{n}(\%)$ & $4(23,5)$ & $16(16,3)$ & $0,469^{\ddagger}$ \\
\hline Edema macular diabético, $\mathrm{n}(\%)$ & $6(35,2)$ & $26(26,5)$ & $0,457^{\ddagger}$ \\
\hline
\end{tabular}

HbA1c: hemoglobina glucosilada; RDNP: retinopatía diabética no proliferativa; RDP: retinopatía diabética proliferativa.

*Significancia estadística $<0,05$.

tU de Mann Whitney.

$\ddagger \mathrm{Ji}$ al cuadrado.

mayoría $(528,74,8 \%)$ tenían diabetes tipo 2 . Solo 43 $(6,1 \%)$ pacientes manifestaron tabaquismo activo en el momento de la evaluación y $381(62,4 \%)$ tenían HTA como comorbilidad.

En la variable de conocimiento de la enfermedad por parte del paciente se encontró que solo 202 (33\%) pacientes manifestaron tener algún grado de conocimiento acerca de la RD y 340 (56\%) sabían qué es la HbA1c. Además, 304 (50\%) asistían nuevamente a la campaña después de haber asistido en algún año anterior. La mediana de los valores de la $\mathrm{HbA} 1 \mathrm{c}$ fue del $7,7 \%$ (RIQ: 6,6-8,4) en toda la muestra, y en 139 (22,7\%) pacientes se encontró alguna complicación asociada a la diabetes, con daño renal asociado en 46 (7,5\%) pacientes. Solo $312(52 \%)$ pacientes habían tenido alguna evaluación de fondo de ojo por oftalmología u optometría en el último año. El diagnóstico adicional más frecuente fue el de catarata, en 96 $(15,7 \%)$ pacientes.

De los pacientes evaluados, en 115 se encontró RD, lo que supone una prevalencia del $18,8 \%$ (Tabla 2). En $44(38,2 \%)$ casos, el diagnóstico de RD se hizo de novo. Se observó un predominio del sexo femenino, con $74(64,3 \%)$ mujeres con diagnóstico de RD. La prevalencia de RD fue mayor en los pacientes con diabetes tipo $1(24,3 \%)$ que en aquellos con diabetes tipo $2(17,9 \%)$. En $82(71,3 \%)$ pacientes con RD se encontró que tenían también HTA. El tipo de RD según la clasificación internacional se encuentra consignado en la tabla 1. La RDP se encontró en 17 (14,7\%) pacientes y la RDNP fue la más frecuente, con 98 pacientes $(85,2 \%)$. De este grupo, la RDNP leve se observó en $57(58,1 \%)$ pacientes, la RDNP moderada en $33(33,6 \%)$ y la RDNP grave en $8(8,1 \%)$. El tiempo de evolución de la diabetes en los pacientes con RD tuvo una mediana de 16,2 años (RIQ: 8-22,7), mientras que en los pacientes sin RD fue de 10 años (RIQ: 4-16). Se encontró una mediana de $\mathrm{HbA1c}$ del 8,2\% (RIQ: 6,9-8,8) en el grupo de pacientes con RD, mientras que en los pacientes sin RD fue del 7,2\% (RIQ: 6,6-8,2). De estos 115 pacientes con RD, $32(27,8 \%)$ presentaban EMD en el momento de la evaluación. Además, 18 (15,6\%) pacientes con $\mathrm{RD}$ tenían algún grado de daño renal. En la tabla 2 se encuentran los datos de los pacientes con RDP y RDNP.

En los 32 casos $(5,2 \%)$ del total de la población en que se encontró EMD, la mediana del tiempo de evolución de la diabetes fue de 11,5 años (RIQ: 6,8-16,7). La mediana de la HbA1c en los pacientes con EMD fue del 8,8\% (RIQ: 7,2-11,8).

El subgrupo de RD que amenaza la visión (RDNP grave, RDP y EMD) estaba constituido por $48(7,8 \%)$ pacientes, de los cuales en $7(14,5 \%)$ no se había hecho el diagnóstico previamente a esta campaña. En este subgrupo, solo $10(20,8 \%)$ pacientes habían recibido algún tipo de tratamiento, ya fuera con fotocoagulación laser retinal o con inyecciones intravítreas de antiangiogénicos. El valor de la $\mathrm{HbA} 1 \mathrm{c}$ tuvo una mediana del 8,6\% (RIQ: 6,8-9). De este subgrupo de alto riesgo para pérdida de la visión, $10(20,8 \%)$ pacientes tenían algún grado de daño renal y 12 (25\%) requirieron remisión a sus aseguradoras para iniciar manejo urgente. Todos los pacientes en este grupo habían sido evaluados previamente en algún momento por oftalmología.

En general, 17 (2,7\%) pacientes requirieron remisión urgente y se solicitaron exámenes complementarios en $41(6,7 \%)$ para ser evaluados por los oftalmólogos de sus entidades aseguradoras.

\section{Discusión}

La RD es una complicación microangiopática de la diabetes, en la que el aumento de la glucemia lleva a una desviación del metabolismo de la glucosa a la vía del sorbitol y al aumento de los productos de la glucosilación avanzada y sus derivados en las células endoteliales y 
pericitos, lo que en últimas conduce a una alteración de la barrera hematorretiniana, con filtración de componentes sanguíneos y formación de microaneurismas y hemorragias $^{6,7}$. Estas alteraciones generan complicaciones de tipo isquémico, lo que estimula la liberación de factores de crecimiento como el factor de crecimiento vascular endotelial, la proliferación vascular, mayores hemorragias y desprendimiento de retina asociado por tracción $n^{7,8}$.

Los estudios poblacionales realizados en países desarrollados muestran que la prevalencia de la RD es del $34,6 \%$ y que la de la RDP alcanza a ser hasta del $7 \%{ }^{9}$. Se dice que el número de personas con RD en el mundo aumentará de 126 millones en el año 2011 a 191 millones en el año $2030^{10}$.

En este estudio, la prevalencia de la RD en la población de pacientes diabéticos evaluados fue del $18,8 \%$, menor que la encontrada en estudios poblacionales en países desarrollados ${ }^{9}$. Sin embargo, las tasas de prevalencia fluctúan entre el $17 \%$ y el $45 \%{ }^{9}$, rango que incluye el valor encontrado en el presente estudio. Al comparar con otros estudios epidemiológicos realizados en América Latina se encuentran valores de prevalencia variados. En Chile, Abuauad, et al. ${ }^{11}$ encontraron una prevalencia del $24 \%$ en una población de 468 pacientes diabéticos. En México, Prado, et al. ${ }^{12}$ obtuvieron una prevalencia mucho mayor, del $71 \%$, en una población de 13,670 pacientes diabéticos en un lapso de 30 años. Otros estudios mostraron una prevalencia similar, como el realizado por Amador, et al. ${ }^{6}$ en Honduras, en el cual se reportó una prevalencia de RD del 19\% en una población de 177 pacientes diabéticos. En Colombia, los casos de RD se han incrementado en los últimos años, pasando de una prevalencia de 12,86 por cada 100.000 habitantes en el año 2009 a 19,76 por cada 100.000 habitantes en $2014^{10}$.

En cuanto al tipo de diabetes, a pesar de que la población de diabéticos de tipo 2 sea mayor, en este estudio se encontró una prevalencia más alta de $\mathrm{RD}$ en los diabéticos de tipo 1 (24,3\%), dato que coincide con otros estudios ${ }^{12}$. En los pacientes en los que se encontró RD, la población de mujeres es mayor, lo que es acorde con lo reportado en la mayoría de los estudios de prevalencia ${ }^{6,12,13}$.

Entre los factores que se han descrito asociados a la $\mathrm{RD}$ se incluyen el tiempo de evolución, los valores de la HbA1c, la HTA y la asociación con la nefropatía diabética $^{14}$. En este estudio se encontró que las medianas del tiempo de duración de la diabetes y del valor de la HbA1c eran mayores en los pacientes con diagnóstico de $\mathrm{RD}$, lo que es comparable con lo descrito en diferentes estudios en todo el mundo $8,15-17$. Presentaban
HTA el $62 \%$ del total de los pacientes diabéticos y específicamente el $71 \%$ de los pacientes con RD. En Cuba, Yáñez, et al. ${ }^{16}$ encontraron HTA en el $42 \%$ de la población diabética, lo que es comparable con los datos de la OMS, que hablan del hallazgo de HTA en el 55\% de los pacientes con diabetes de tipo $2^{14}$. Así mismo, encontramos que el $13 \%$ de los pacientes con RD presentaban daño renal como complicación de la enfermedad, frente a solo el 4,4\% de los pacientes diabéticos sin RD. Estos datos son similares a los encontrados en un estudio con 472 pacientes diabéticos en $\mathrm{Cuba}^{16}$, en el que hallaron nefropatía diabética en el $3,7 \%$ de los pacientes $\sin \mathrm{RD}$ y en el $10 \%$ de los pacientes con RD.

El EMD es la causa principal de pérdida de visión en los pacientes diabéticos ${ }^{17}$, por lo cual es importante reconocer su prevalencia en las diferentes poblaciones, y más ahora con la mayor accesibilidad a exámenes diagnósticos como la tomografía de coherencia óptica, de manera que pueda detectarse de forma temprana y se le pueda dar un manejo adecuado para mejorar el pronóstico. En este estudio se encontró EMD en el 5,2\% de la población diabética, lo que se encuentra en el rango esperado según la prevalencia mundial, que varía según la región ${ }^{18}$, con prevalencias del $3,8 \%$ en los Estados Unidos de Norteamérica ${ }^{15}$, valores tan bajos como el 1,8\% en países como Chile ${ }^{19}$ y valores tan elevados como el $15 \%$ en Turquía ${ }^{17}$.

Se ha descrito una relación no lineal entre los valores de la HbA1c y el EMD ${ }^{15}$, y esto se encuentra asociado a una duración más prolongada de la diabetes. En el presente estudio también se halló que, en ese grupo de pacientes con EMD, tanto el tiempo de evolución como los valores de la HbA1c fueron mayores en comparación con los pacientes sin EMD.

Para el grupo de pacientes con RD que amenaza la visión, en el mundo se ha visto un aumento de la prevalencia a medida que aumentan los pacientes diabéticos, e incluso se ha encontrado que hasta en el $11,1 \%$ de estos casos no se había hecho el diagnóstico de RD previamente ${ }^{5}$, lo que se describe asociado a fallas o escasez de programas de tamización. En el presente estudio, en el $14 \%$ de los pacientes con RD que amenaza la visión no se había hecho diagnóstico previo de $\mathrm{RD}$, en concordancia con las cifras descritas, siendo incluso un poco mayores. La prevalencia encontrada de RD que amenaza la visión fue del $7,8 \%$, valor comparable con la prevalencia en diferentes regiones del mundo, como Suecia, donde Gustafsson y Hellgren ${ }^{20}$ encontraron una prevalencia del 6,8\%. La incidencia de la RD que amenaza la visión, como se describió, guarda relación con el tiempo de evolución de la 
diabetes, y según un estudio realizado en Liverpool pasa del $0,3 \%$ en el primer año al $3.9 \%$ en el quinto año de tamización ${ }^{21}$.

Si se tiene en cuenta que la RD tiene el potencial de ser la primera causa de pérdida visual y ceguera en todo el mundo, y que el $80 \%$ de los casos de ceguera por RD son prevenibles con un diagnóstico y un tratamiento tempranos, sobre todo en los pacientes con RD que amenaza la visión, es evidente el efecto tan relevante que tienen los programas de tamización efectivos y controlados sobre este dramático problema de salud pública, con el objetivo de tener una mejor planeación de las necesidades y de los costos asociados al tratamiento de estos pacientes, y así estimar de forma precisa la magnitud de la RD, del EMD y de la $\mathrm{RD}$ que amenaza la visión y sus factores de riesgo en las diferentes regiones latinoamericanas.

\section{Conclusiones}

La prevalencia de la RD en la población estudiada es comparable con la encontrada en algunos de los estudios realizados en América Latina. El valor de la HbA1c y el tiempo de evolución de la diabetes son determinantes en la aparición de EMD y la pérdida de visión. A pesar de las limitaciones de este estudio, por ser transversal, basar gran parte de su información en encuestas y la heterogeneidad de los pacientes, consideramos que aporta datos epidemiológicos locales de interés y además refuerza la importancia de los programas de tamización.

\section{Agradecimientos}

Agradecimiento especial a todo el equipo de trabajo de la campaña "No más ciegos por diabetes" que se realiza cada año y del cual hacen parte retinólogos, oftalmólogos, residentes, estudiantes de medicina y todos los colaboradores del Hospital San Vicente Fundación de Medellín.

\section{Conflicto de intereses}

Los autores declaran no tener ningún conflicto de intereses.

\section{Responsabilidades éticas}

Protección de personas y animales. Los autores declaran que para esta investigación no se han realizado experimentos en seres humanos ni en animales.

Confidencialidad de los datos. Los autores declaran que han seguido los protocolos de su centro de trabajo sobre la publicación de datos de pacientes.

Derecho a la privacidad y consentimiento informado. Los autores han obtenido el consentimiento informado de los pacientes y/o sujetos referidos en el artículo. Este documento obra en poder del autor de correspondencia.

\section{Bibliografía}

1. Preferred Practice Pattern® Guidelines. Diabetic retinopathy. American Academy of Ophthalmology Retina/Vitreous Panel. 2017. Disponible en: www.aao.org/ppp

2. Wu L, Fernández-loaiza $P$, Sauma J, Hernandez-Bogantes E, Masis M, Fernandez-Loaiza $P$, et al. Classification of diabetic retinopathy and diabetic macular edema. World J Diabetes. 2013;4:290-4.

3. Guías clínicas para el manejo de la patología ocular del diabético. Consejo Internacional de Oftalmología. 2017. Disponible en: http://www.icoph.org/

4. Williams R, Airey M, Baxter H, Forrester J, Kennedy-Martin T. Epidemiology of diabetic retinopathy and macular oedema: a systematic review. Eye. 2004;18:963-83.

5. Huang OS, Tay WT, Ong PG, Sabanayagam C, Cheng C, Tan GS, et al. Prevalence and determinants of undiagnosed diabetic retinopathy and vision-threatening retinopathy in a multiethnic Asian cohort: the Singapore Epidemiology of Eye Diseases (SEED) study. Br J Ophthalmol Publ. 2015;99:1614-21.

6. Amador-Rosa ME, García JA, Amador-Rosa ME. Prevalencia de retinopatía diabética en el Instituto Nacional del Diabético de Honduras, en el periodo febrero-julio 2016. Arch Med. 2016;12:1-7.

7. Zhang K, Ferreyra HA, Grob S. Diabetic retinopathy: genetics and etiologic mechanisms. En: Ryan SJ, editor. Retina, 5th ed. Philadelphia: Elsevier Saunders; 2013. p. 925-39.

8. Vallejo EV, Rodríguez FJ. Epidemiología de la retinopatía diabética y su relación con la diabetes. Rev Colomb Endocrinol Diabetes Metab. 2016;3:12-5.

9. Yau JWY, Rogers SL, Kawasaki R. Global prevalence and major risk factors of diabetic retinopathy. Diabetes Care. 2012;35:556-64.

10. Análisis de situación de salud visual en Colombia. Ministerio de Salud de Colombia. 2016. Disponible en: https://www.minsalud.gov.co

11. Abuauad $S$, Guzmán $P$, Urzúa $C$. Prevalencia de retinopatía diabética y edema macular en población diabética del CESFAM Cordillera Andina de Los Andes. Rev Chil Salud Publica. 2014;18:81-6.

12. Prado-Serrano A, Guido-Jiménez DMA, Camas-Benítez DJT. Prevalencia de retinopatía diabética en población mexicana. Rev Mex Oftalmol. 2009;83:261-6.

13. Chibás AR, Lozada IR. Caracterización de retinopatía diabética en pacientes del Policlínico Universitario "Asdrúbal López". Rev Inf Cient. 2009;61:1-11.

14. World Health Organization. Prevention of blindness from diabetes mellitus: report of a WHO consultation. Geneva: WHO; 2006

15. Varma R, Bressler NM, Doan Q V, Gleeson M, Danese M, Bower JK, et al. Prevalence of and risk factors for diabetic macular edema in the United States. JAMA Ophthalmol. 2014;132:1334-40.

16. Yáñez B, Murillo JP, Arbañil $H$. Diabetic retinopathy: prevalence and associated risk factors. Revista Médica Carrionica. 2016;3:3-14.

17. Acan D, Calan M, Er D, Arkan T, Kocak N, Bayraktar F, et al. The prevalence and systemic risk factors of diabetic macular edema: a cross-sectional study from Turkey. BMC Ophthalmol. 2018;18:1-8.

18. Lee R, Wong TY, Sabanayagam C. Epidemiology of diabetic retinopathy, diabetic macular edema and related vision loss. Eye Vis. 2015;2:1-25.

19. Soto $A$, Cruz $C$. Retinopatía diabética y edema macular diabético: prevalencia en una población de atención primaria de Antofagasta. Rev Chil Salud Pública. 2018;22:155-60.

20. Gustafsson S, Hellgren K-J. Sight-threatening diabetic retinopathy in a Swedish County. Orebro University; 2017. Disponible en: http://urn.kb.se/ resolve?urn=urn:nbn:se:oru:diva-66795

21. Younis N, Broadbent DM, Vora JP, Harding SP. Incidence of sight-threatening retinopathy in patients with type 2 diabetes in the Liverpool Diabetic Eye Study: a cohort study. Lancet. 2003;361:195-200. 